\title{
KALMAN TRACKING OF TIME-VARYING CHANNELS IN WIRELESS MIMO-OFDM SYSTEMS
}

\author{
Dieter Schafhuber, Gerald Matz, and Franz Hlawatsch \\ Institute of Communications and Radio-Frequency Engineering, Vienna University of Technology \\ Gusshausstrasse 25/389, 1040 Vienna, Austria (Europe) \\ phone: +43 158801 38973, fax: +43 158801 38999, email: Dieter.Schafhuber@ieee.org \\ web: www.nt.tuwien.ac.at/dspgroup/dschafhu.htm
}

\begin{abstract}
The performance of coherent MIMO-OFDM systems critically depends on the availability of accurate channel estimates. In wireless communications, the MIMO channel is time-varying and thus has to be tracked by the receiver. In this paper, we develop an extended Kalman filter technique for pilot symbol assisted MIMO-OFDM channel tracking. Our tracking scheme is able to exploit spatial correlations of the channel. It includes on-line estimation of the channel's state-space parameters and hence does not require any prior knowledge. Simulations using measured channels demonstrate the excellent performance of our channel tracking scheme.
\end{abstract}

\section{INTRODUCTION}

Multi-input multi-output (MIMO) wireless communication systems using multiple transmit and receive antennas can yield improved link reliability through spatial diversity and increased data rate through multiplexing techniques $[1,2]$. MIMO-OFDM is a promising modulation scheme for broadband MIMO communications over delay-spread channels $[3,4]$. For MIMO-OFDM, several pilot symbol assisted channel estimation techniques have been proposed for providing the receiver with the channel state information required for large diversity and multiplexing gains [5-8]. These techniques presuppose (partial) knowledge of the channel statistics. Estimation of the channel statistics is difficult and needs to be repeated periodically because the channel statistics change over time. In [9], we proposed an adaptive channel tracking method that does not require any prior knowledge. However, this method does not exploit spatial correlations of the MIMO channel.

In this paper, we present a novel adaptive channel tracking scheme based on the extended Kalman filter. This scheme is able to exploit spatial correlations of the channel. It includes on-line estimation of the channel's state-space parameters (state-transition matrix and noise variances) and hence does not require any prior knowledge.

Previous work proposing Kalman filters for MIMO channel estimation includes $[10,11]$. However, [10] does not assume OFDM modulation and does not consider estimation of the channel statistics. OFDM modulation is assumed in [11], but again the channel statistics are supposed to be known. Neither of the two schemes uses the extended Kalman filter.

This paper is organized as follows. Section 2 reviews the MIMOOFDM system model. In Section 3, we present the overall channel

\footnotetext{
Funding by FWF grant P15156.
}

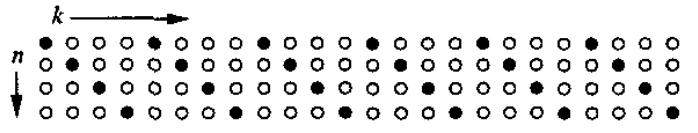

Figure 1: Location of pilot symbols $(\bullet)$ and data symbols $(0)$ for one transmit antenna and $K=24, P=6$, and $S=4$.

tracking scheme. The application of the extended Kalman filter is described in Section 4, and on-line estimation of the relevant noise variances is discussed in Section 5. Finally, in Section 6 we assess the performance of our method through numerical simulations.

\section{MIMO-OFDM SYSTEM MODEL}

We first discuss the MIMO-OFDM system model on which our channel tracking technique will be based (cf. [9]).

Modulator. We consider a MIMO-OFDM system with $K$ subcarriers, $M_{\mathrm{T}}$ transmit antennas, and $M_{\mathrm{R}}$ receive antennas. The symbol vectors are $\mathbf{a}_{n, k}=\left[a_{n, k}^{(1)} \cdots a_{n, k}^{\left(M_{\mathrm{T}}\right)}\right]^{T}, n \in \mathbb{Z}, k=0, \ldots, K-1$, where $a_{n, k}^{(i)}$ denotes the symbol transmitted at symbol time $n$, subcarrier $k$, and antenna $i$. The $n$th OFDM symbol vector $s_{n}[m]$ is obtained by applying an inverse discrete Fourier transform (IDFT) to the $\mathbf{a}_{n . k}$ and prepending a cyclic prefix of length $L_{\mathrm{cp}}$ :

$$
\mathbf{s}_{n}[m]= \begin{cases}\frac{1}{{\sqrt{K M_{T}}}_{k=0}^{K-1} \mathbf{a}_{n, k} e^{j 2 m k / K},} & m=-L_{\mathrm{cp}} \ldots, K-1 \\ 0, . & \text { else. }\end{cases}
$$

Thus, the duration of each OFDM symbol is $N=K+L_{\mathrm{cp}}$ samples. The overall baseband transmit signal is $\mathbf{s}[m]=n=-\mathbf{s}_{n}[m-n N]$.

Pilot symbols. At locations $(n, k) \in \mathscr{P}$, pilot symbols known to the receiver are transmitted. We will use the pilot location set [12]

$$
\mathscr{P}=\{(n . k) \mid n \in \mathbb{Z} . k=i S+(n \bmod S) \text { with } i=0, \ldots, P-1\},
$$

where $P$ is the number of pilots per OFDM symbol and $S=K / P$ (assumed an integer) is the distance of the pilots. An example is shown in Fig. 1. We propose to construct the pilot symbols as [9]

$$
\mathbf{a}_{n, k}=w_{n . k} \mathbf{p}_{n}
$$

where $w_{n, k} \in\{1,-1\}$ is a pseudo-noise sequence (for later convenience, we define $w_{n, k} \triangleq 0$ for $\left.(n, k) \notin \mathscr{P}\right)$ and $\mathbf{p}_{n}$ is a spatial pilot 


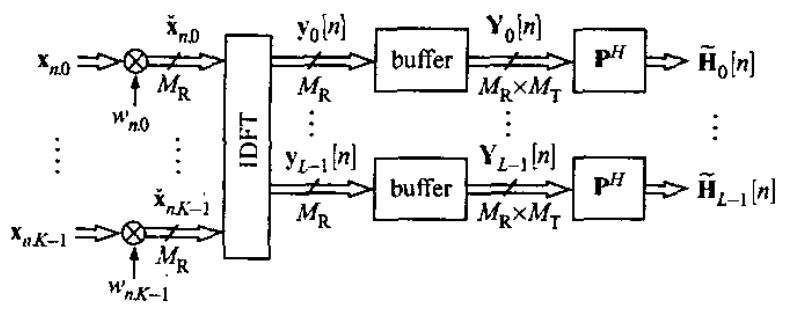

Figure 2: Preprocessing stage of the adaptive channel tracking scheme.

spreading vector that is periodic with period $M_{\mathrm{T}}$, i.e., $\mathbf{p}_{n+M_{\tau}}=\mathbf{p}_{n}$. Thus, it suffices to specify $\mathbf{P} \triangleq \frac{1}{\sqrt{M_{\mathrm{T}}}}\left[\mathbf{p}_{1} \cdots \mathbf{p}_{M_{\mathrm{T}}}\right]$, which we assume to be orthogonal, i.e., $\mathbf{P} \mathbf{P}^{H}=\mathbf{P}^{H} \mathbf{P}=\mathbf{1}$. This implies that the pilot sequences transmitted from different antennas are orthogonal. A convenient choice for $\mathbf{P}$ is the $M_{\mathrm{T}} \times M_{\mathrm{T}}$ DFT matrix (e.g., for $M_{\mathrm{T}}=2$ this gives $\mathbf{p}_{1}=\left[\begin{array}{ll}1 & 1\end{array}\right]^{T}$ and $\mathbf{p}_{2}=\left[\begin{array}{ll}1 & -1\end{array}\right]^{T}$ ).

Channel. We assume a fading multipath MIMO channel that is characterized by its $M_{\mathrm{R}} \times M_{\mathrm{T}}$ baseband impulse response matrix $\mathbf{H}[m . l]$. The $M_{\mathrm{R}} \times !$ receive signal vector is given by

$$
\mathbf{r}[m]={ }_{l=0}^{L-1} \mathbf{H}[m, l] \mathbf{s}[m-l]+[m] .
$$

where $L$ is the number of channel taps and $[m]$ is stationary white noise with complex normal distribution $\mathscr{C}_{\mathcal{C}} \mathscr{W}^{\prime}\left(0,{ }^{2} \mathrm{I}\right)$. We assume $L \leq L_{\mathrm{cp}}+1$ so that intersymbol interference is avoided. We furthermore assume that the channel's fading rate is limited to the maximum normalized Doppler frequency max

Demodulator. The received signal $\mathbf{r}[m]$ is demodulated by discarding the cyclic prefix and computing a normalized DFT, i.e.,

$$
\mathbf{x}_{n \cdot k}=\frac{1}{\sqrt{K}}_{m=0}^{K-1} \mathbf{r}[n N+m] e^{-j 2 k m / K} .
$$

If $N \max \ll 1$, the impulse response $\mathbf{H}[m, l]$ varies negligibly within one symbol period. The input-output relation of the overall OFDM system is then obtained as $[3,4]$

$$
\mathbf{x}_{n, k}=\check{\mathbf{H}}_{n, k} \mathbf{a}_{n, k}+\check{ }_{n, k},
$$

with the time/frequency-domain channel coefficients

$$
\check{\mathbf{H}}_{n, k} \triangleq{\frac{1}{\sqrt{M_{\mathrm{T}}}}}_{l=0}^{L-1} \mathbf{H}[n N, I] e^{-j 2 k l / K}
$$

and the noise $\quad{ }_{n, k} \triangleq \frac{1}{\sqrt{K}} \underset{m=0}{K-1}[n N+m] e^{-j 2 \quad k m / K}$.

\section{CHANNEL TRACKING PROCEDURE}

The proposed channel tracking procedure consists of three steps. First, a preprocessing step (cf. [9]) compensates the frequency-dependent factor of the pilot symbols and performs an IDFT to obtain a simple input-output relation in the time/delay domain. Second, the extended Kalman filter $[13,14]$ is applied to track the (subsampled) channel tap matrices. Finally, a DFT postprocessing step (cf. [9]) yields the estimated time/frequency-domain channel coefficient matrices. In the following, we describe these steps in more detail.

Preprocessing. The preprocessing (see Fig. 2) is a preparatory stage that enables a reduced-complexity implementation of channel tracking in the time/delay domain. The complexity is reduced because the number of coefficients in the time/delay domain is much smaller than in the time/frequency domain $(L \ll K)$.

We first compensate the subcarrier-dependent pilot symbol factor $w_{n, k}$ by forming

$$
\check{\mathbf{x}}_{n, k} \triangleq w_{n, k} \mathbf{x}_{n, k} .
$$

Because $\mathbf{a}_{n, k}=w_{n, k} \mathbf{p}_{n}$ with $w_{n, k} \in\{1,-1\}$ for $(n, k) \in \mathscr{P}$ and $w_{n, k}=0$ for $(n, k) \notin \mathscr{P}$, we obtain

$$
\check{\mathbf{x}}_{n, k}= \begin{cases}\check{\mathbf{H}}_{n, k} \mathbf{p}_{n}+\check{\mathbf{z}}_{n, k}, & (n, k) \in \mathscr{P}, \\ 0, & \text { else, }\end{cases}
$$

with $\check{\mathbf{z}}_{n, k} \triangleq w_{n, k} \quad{ }_{n . k}$. Assuming $P \geq \dot{L}$ to avoid aliasing, we can now transform $\check{\mathbf{x}}_{n, k}$ into the time/delay domain (cf. (1)) by the IDFT

$$
\mathbf{y}_{l}[n] \triangleq{\frac{\sqrt{M_{\mathrm{T}}}}{P}}_{k=0}^{K-1} \check{\mathbf{x}}_{n \cdot k} e^{j 2 l k / K} .
$$

Note that this IDFT involves only $P$ nonzero $\check{x}_{n, k}$ per symbol period. We obtain

$$
\mathbf{y}_{l}[n]= \begin{cases}\mathbf{H}[n N, l] \mathbf{p}_{n}+\dot{\mathbf{z}}_{l}[n], & l=0, \ldots, L-1, \\ \overrightarrow{\mathbf{z}}_{l}[n], & l=L, \ldots, P-1,\end{cases}
$$

with $\check{z}_{l}[n] \triangleq \frac{\sqrt{M_{T}}}{P} \quad{ }_{k=0}^{K-1} \check{z}_{n, k} e^{j 2} \quad l k / K$. The $\mathbf{y}_{l}[n]$ for delays $l \geq L$ will be discarded in the subsequent channel tracking since they consist only of noise. If $L$ is not exactly known, the worst-case choice $L=$ $L_{\mathrm{cp}}+1$ can be used.

We next assume $M_{\mathrm{T}} N \max \ll 1$, i.e., that the channel varies negligibly within $M_{\mathrm{T}}$ symbol periods. Thus, by idealization, $\mathbf{H}[(n+$ i) $N, l]=\mathbf{H}[n N, l]$ for $i=1, \ldots, M_{\mathrm{T}}-1$. For the $M_{\mathrm{R}} \times M_{\mathrm{T}}$ matrix $\mathbf{Y}_{l}[n] \triangleq\left[\mathbf{y}_{l}\left[n M_{\mathrm{T}}\right] \mathbf{y}_{l}\left[n M_{\mathrm{T}}+1\right] \cdots \mathbf{y}_{l}\left[(n+1) M_{\mathrm{T}}-1\right]\right]$, we then obtain

$$
\mathbf{Y}_{l}[n]=\mathbf{H}_{l}[n] \mathbf{P}+\widetilde{\mathbf{Z}}_{l}[n]
$$

with $\mathbf{H}_{l}[n] \triangleq \mathbf{H}\left[n M_{\mathrm{T}} N, l\right]$ and $\tilde{\mathbf{Z}}_{l}[n] \triangleq\left[\tilde{\mathbf{z}}_{l}\left[n M_{\mathrm{T}}\right] \mathbf{\mathbf { z }}_{l}\left[n M_{\mathrm{T}}+1\right] \cdots \dot{\mathbf{z}}_{l}[(n+\right.$ 1) $\left.\left.M_{\mathrm{T}}-1\right]\right]$. Note that (2) involves a decimation of $\mathbf{H}[m, l]$ with respect to $m$ by the factor $M_{\mathrm{T}}$.

The last preprocessing step is a multiplication of $\mathbf{Y}_{l}[n]$ by $\mathbf{P}^{H}$, which corresponds to a correlation with the spatial pilot sequences. Using the orthogonality of the pilot matrix $\mathbf{P}$, we obtain the simple "signal-plus-noise" relation

$$
\widetilde{\mathbf{H}}_{l}[n] \triangleq \mathbf{Y}_{l}[n] \mathbf{P}^{H}=\mathbf{H}_{l}[n]+\mathbf{Z}_{l}[n],
$$

where the elements of all matrices $\mathbf{Z}_{l}[n] \triangleq \tilde{\mathbf{Z}}_{l}[n] \mathbf{P}^{H}$ are i.i.d. and distributed as $\mathscr{C} \mathscr{N}\left(0,{ }_{z}^{2}\right)$ with ${ }_{z}^{2}={ }^{2} / P$.

Channel state-space model. The proposed channel tracking procedure calculates channel estimates $\widehat{\mathbf{H}}_{l}[n]$ from the "pre-estimates" $\tilde{\mathbf{H}}_{l}[n]$ in (3). In what follows, it will be convenient to use the channel vector $h_{l}[n] \triangleq \operatorname{vec}\left\{\mathbf{H}_{l}[n]\right\}$ of size $M \triangleq M_{\mathrm{T}} M_{\mathrm{R}}$ that is obtained by stacking all columns of the channel matrix $\mathbf{H}_{l}[n]$. 
The Kalman filter tracking scheme is based on the following statespace model for the channel's temporal evolution:

$$
\mathbf{h}_{l}[n+1]=\mathbf{F h}_{l}[n]+\mathbf{u}_{l}[n],
$$

with the $M \times M$ state-transition matrix $\mathbf{F}$ and the $M \times 1$ innovations noise vector $\mathrm{u}_{l}[n]$. The recursion (4) is started at time $n=0$. The matrix $\mathbf{F}$ models the spatio-temporal correlations of the channel; note that the same $\mathrm{F}$ is used for all delay indices $l$. We assume that $\mathbf{u}_{l}[n]$ and $\mathbf{h}_{l}[0]$ are mutually uncorrelated, zero-mean, complex Gaussian vectors with correlation functions $\mathrm{E}\left\{\mathbf{u}_{j}[n] \mathbf{u}_{i}^{H}\left[n^{\prime}\right]\right\}=$ ${ }_{u}^{2}{ }_{n-n^{\prime}}{ }_{l-l^{\prime}} \mathbf{I}$ and $\mathrm{E}\left\{\mathbf{h}_{l}[0] \mathbf{h}_{\eta^{H}}^{H}[0]\right\}=l_{-l^{\prime}} \mathbf{I}$, respectively.

The state-space model equation (4) is complemented by the vectorized version of (3),

$$
\tilde{\mathbf{h}}_{l}[n]=\mathbf{h}_{l}[n]+\mathbf{z}_{l}[n]
$$

where $\tilde{\mathbf{h}}_{l}[n] \triangleq \operatorname{vec}\left\{\tilde{\mathbf{H}}_{l}[n]\right\}$ and $\mathbf{z}_{l}[n] \triangleq \operatorname{vec}\left\{\mathbf{Z}_{l}[n]\right\} ;$ note that $\mathbf{z}_{l}[n]$ is a zero-mean, complex Gaussian vector with correlation function $\mathrm{E}\left\{\mathrm{z}_{l}[n] \mathbf{z}_{\gamma^{\prime}}^{H}\left[n^{\prime}\right]\right\}={\underset{z}{2}}_{n-n^{\prime}}{ }_{1-p^{\prime}} \mathrm{I}$ where ${ }_{z}^{2}=2 / P$.

If the parameters $F, \frac{2}{u}$, and ${ }_{z}^{2}$ were known, the Kalman filter equations [14] could be directly applied for channel tracking, i.e., for calculating channel estimates $\hat{\mathbf{h}}_{l}[n]=\operatorname{vec}\left\{\hat{\mathbf{H}}_{l}[n]\right\}$. However, these parameters are unknown. The estimation of $\mathbf{F}$ will be part of the extended Kalman filter described in Section 4. The estimation of ${ }_{u}^{2}$ and ${ }_{z}^{2}$ will be discussed in Section 5 .

Postprocessing. Finally, the estimated channel tap matrices $\widehat{\mathbf{H}}_{l}[n]$ are transformed back into the time/frequency domain as (cf. (1))

$$
\widehat{\mathbf{H}}_{n M_{\mathrm{T}}, k}={\frac{1}{\sqrt{M_{\mathrm{T}}}}}_{l=0}^{L-1} \widehat{\mathbf{H}}_{l=0}[n] e^{-j 2 k l / K} .
$$

The missing intermediate channel coefficients are recovered through the trivial interpolation $\widehat{\mathbf{H}}_{n M_{\mathrm{T}}+i, k}=\widehat{\mathbf{H}}_{n M_{\mathrm{T}}, k}, i=1, \ldots, M_{\mathrm{T}}-1$ (this is justified by our assumption that the channel varies negligibly during $M_{\mathrm{T}}$ symbol periods).

\section{EXTENDED KALMAN FILTER}

To include on-line estimation of the state-transition matrix $\mathbf{F}$ in our channel tracking scheme, we will now augment the state vector (cf. (4)) by vec $\{\mathbf{F}\}$. This leads to a nonlinear state-space model. We will then use the extended Kalman filter (EKF) $[13,14]$ to linearize the problem and estimate the augmented state vector. We thereby obtain a method for simultaneous channel tracking and estimation of $\mathbf{F}$. For the formulation of the EKF, the noise variances ${ }_{4}^{2}$ and ${ }_{2}^{2}$ are assumed known; their estimation will be discussed in Section 5 .

Augmented state space. We shall use the following combined and augmented form of the state-space equations (4):

$$
\mathbf{x}[n+1]=g(\mathbf{x}[n])+\mathbf{u}[n] .
$$

Here, the $(L+M) M \times 1$ augmented state vector and corresponding nonlinear state-transition function are respectively given by

$$
\mathbf{x}[n] \triangleq\left[\begin{array}{c}
\mathbf{h}_{0}[n] \\
\vdots \\
\mathbf{h}_{L-1}[n] \\
\operatorname{vec}\{\mathbf{F}\}
\end{array}\right] \quad \text { and } \quad g(\mathbf{x}[n]) \triangleq\left[\begin{array}{c}
\mathbf{F} \mathbf{h}_{0}[n] \\
\vdots \\
F_{\mathbf{h}_{L-1}[n]} \\
\operatorname{vec}\{\mathbf{F}\}
\end{array}\right]
$$

and the augmented innovations noise vector is given by $\mathbf{u}[n]=$

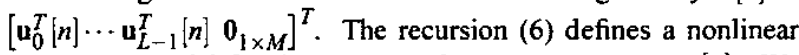
state-space model for the augmented state-space vector $\mathbf{x}[n]$. We furthermore combine the equations (5) as

$$
\tilde{\mathbf{h}}[n]=\mathbf{Q} \mathbf{x}[n]+\mathbf{z}[n],
$$

with the $L M \times 1$ vectors $\overline{\mathbf{h}}[n] \triangleq\left[\tilde{\mathbf{h}}_{0}^{T}[n] \cdots \tilde{\mathbf{h}}_{L-1}^{T}[n]\right]^{T}$ and $\mathbf{z}[n]$ $\triangleq\left[\mathbf{z}_{0}^{T}[n] \cdots \mathbf{z}_{L-1}^{T}[n]\right]^{T}$ and the $L M \times(L+M) M$ matrix $\mathbf{Q} \triangleq$ $\left[\mathbf{I}_{L M} \mathbf{0}_{L M \times M^{2}}\right]$.

For estimation of $\mathbf{x}[n]$, we will apply the EKF equations in measurement update and time update form [14]. In what follows,

$$
\hat{\mathbf{x}}[n \mid m]=\left[\begin{array}{c}
\hat{\mathbf{h}}_{0}[n \mid m] \\
\vdots \\
\hat{\mathbf{h}}_{L-1}[n \mid m] \\
\operatorname{vec}\{\hat{\mathbf{F}}[n \mid m]\}
\end{array}\right]=\left[\begin{array}{c}
\hat{\mathbf{h}}[n \mid m] \\
\operatorname{vec}\{\widehat{\mathbf{F}}[n \mid m]\}
\end{array}\right]
$$

denotes an estimate of $\mathbf{x}\{n\}$ using all measurements until time $m$, and the $(L+M) M \times(L+M) M$ matrix $\Pi[n \mid m]$ denotes an estimate of the corresponding error covariance matrix $\mathrm{E}\{(\mathbf{x}[n]-\hat{\mathbf{x}}[n \mid m])(\mathbf{x}[n]-$ $\left.\hat{\mathbf{x}}[n \mid m])^{H}\right\}$.

Measurement update. Within the measurement update step, $\hat{\mathbf{x}}[n \mid n]$ (the "filtered estimate") is calculated as

$$
\hat{\mathbf{x}}[n \mid n]=\hat{\mathbf{x}}[n \mid n-1]+\mathbf{K}[n](\tilde{\mathbf{h}}[n]-\hat{\mathbf{h}}[n \mid n-1]) .
$$

with the $(L+M) M \times L M$ Kalman gain matrix

$$
\mathbf{K}[n]=\mathbf{\Pi}[n \mid n-1] \mathbf{Q}^{H}\left(\mathbf{Q} \Pi[n \mid n-1] \mathbf{Q}^{H}+{ }_{2}^{2} \mathbf{I}_{L M}\right)^{-1} .
$$

The second part of the measurement update step is calculation of

$$
\Pi[n \mid n]=\left(\mathbf{I}_{(L+M) M}-\mathbf{K}[n] \mathbf{Q}\right) \mathbf{\Pi}[n \mid n-1\} .
$$

Calculation of $\hat{\mathbf{x}}[n \mid n-1]$ and $\mathbf{I}[n \mid n-1]$ will be discussed next.

Time update. Within the time update step, $\hat{\mathbf{x}}[n+1 \mid n]$ (the "predicted estimate") is calculated as

$$
\hat{\mathbf{x}}[n+1 \mid n]=g(\hat{\mathbf{x}}[n \mid n])=\left[\begin{array}{c}
\widehat{\mathbf{F}}[n \mid n] \hat{\mathbf{h}}_{0}[n \mid n] \\
\vdots \\
\widehat{\mathbf{F}}[n \mid n] \hat{\mathbf{h}}_{L-1}[n \mid n] \\
\operatorname{vec}\{\widehat{\mathbf{F}}[n \mid n]\}
\end{array}\right] .
$$

The second part of the time update step is calculation of

$$
\Pi[n+1 \mid n]=\mathbf{G}[n] \mathbf{\Pi}[n \mid n] \mathbf{G}^{H}[n]+{ }_{2}^{2}\left[\begin{array}{cc}
\mathbf{l}_{L M} & \mathbf{0}_{L M \times M^{2}} \\
\mathbf{0}_{M \times L M} & \mathbf{0}_{M N^{2} \times M^{2}}
\end{array}\right],
$$

with the $(L+M) M \times(L+M) M$ matrix (resulting from linearization of $g(\mathbf{x}))$

$$
\begin{aligned}
\mathbf{G}[n] & \left.\triangleq \frac{g(\mathbf{x})}{\mathbf{x}}\right|_{\mathbf{x}=\hat{\mathbf{x}}[n \mid n]} \\
& =\left[\begin{array}{ccccc}
\widehat{\mathbf{F}}[n \mid n] & \mathbf{0}_{M \times M} & \cdots & \mathbf{0}_{M \times M} & \hat{\mathbf{h}}_{0}^{T}[n \mid n] \otimes \mathbf{I}_{M} \\
\mathbf{0}_{M \times M} & \widehat{\mathbf{F}}[n \mid n] & \cdots & \mathbf{0}_{M \times M} & \hat{\mathbf{h}}_{1}^{T}[n \mid n] \otimes \mathbf{l}_{M} \\
\vdots & \vdots & \ddots & \vdots & \vdots \\
\mathbf{0}_{M \times M} & \mathbf{0}_{M \times M} & \cdots & \widehat{\mathbf{F}}[n \mid n] & \hat{\mathbf{h}}_{L-1}^{T}[n \mid n] \otimes \mathbf{l}_{M} \\
\mathbf{0}_{M^{2} \times M} & \mathbf{0}_{M^{2} \times M} & \cdots & \mathbf{0}_{M^{2} \times M} & \mathbf{I}_{M^{2}}
\end{array}\right],
\end{aligned}
$$


where $\otimes$ denotes the Kronecker product [15]. Note that $\hat{\mathbf{h}}_{l}[n \mid n]$, $\hat{\mathbf{F}}[n \mid n]$, and $\Pi[n \mid n]$ are the result of the measurement update equations (9) and (10).

Recursive channel tracking algorithm. The measurement update and time update equations constitute a recursive algorithm for estimating/tracking the channel tap matrices $\mathbf{H}_{l}[n]$ via the identity $\operatorname{vec}\left\{\hat{\mathbf{H}}_{l}[n]\right\} \triangleq \hat{\mathbf{h}}_{l}[n \mid n]$ (cf. (8)). This recursive algorithm starts with the measurement update step at time $n=1$, which is initialized by

$$
\hat{\mathbf{x}}[1 \mid 0]=\left[\begin{array}{c}
\tilde{\mathbf{h}}_{0}[0] \\
\vdots \\
\tilde{\mathbf{h}}_{L-1}[0] \\
\operatorname{vec}\left\{\mathbf{I}_{M}\right\}
\end{array}\right] \quad \text { and } \quad \Pi[1 \mid 0]=\mathbf{I}_{(L+M) M} .
$$

Note that the algorithm also estimates/tracks the state-transition matrix $\mathbf{F}$ via $\widehat{\mathbf{F}}[n \mid n]$. The estimates $\widehat{\mathbf{F}}[n \mid n]$ provide information about the spatio-temporal correlations of the channel.

\section{ESTIMATION OF NOISE VARIANCES}

We will now discuss on-line estimation of the noise variances ${ }_{u}^{2}$ and ${ }_{z}^{2}$ using the approach proposed in $[16,17]$.

Innovations noise variance. Direct estimation of $\underset{n}{2}$ is impossible since the innovations noise $\mathbf{u}_{l}[n]=\mathbf{h}_{l}[n+1]-\mathbf{F} \mathbf{h}_{l}[n]$ (cf. (4)) cannot be observed. However, we can define an estimate of $u_{l}[n]$ by

$$
\hat{\mathbf{u}}_{l}[n]=\hat{\mathbf{h}}_{l}[n \mid n]-\hat{\mathbf{F}}[n \mid n] \hat{\mathbf{h}}_{l}[n-1 \mid n-1] .
$$

where the estimates $\hat{\mathbf{h}}_{l}[n \mid n]$ and $\hat{\mathbf{F}}_{[}[n \mid n]$ are elements of the vector $\hat{\mathbf{x}}[n \mid n]$ (see (8)) that has been calculated in (9). The innovations noise variance ${ }_{u}^{2}$ can then be estimated at time $n$ by

$$
\widehat{\frac{2}{u}}[n]=\frac{1}{\operatorname{LnM}}_{l=0}^{L-1} n\left\|\hat{\mathbf{u}}_{l}[m]\right\|^{2} .
$$

If temporal variations of ${ }_{u}^{2}$ are to be tracked, the average of $\left.\| \hat{\mathbf{u}}_{l}[m\}\right] \|^{2}$ over the entire past (time interval $[1, n]$ ) can be replaced by a local average using a sliding window. To avoid excessively small values of $\frac{2}{4}[n]$ that would result in slow channel tracking of the EKF, a limitation according to $\max \left\{\widehat{\frac{2}{u}}[n],\right\}$ can be employed. (We used $=10^{-4}$ in our simulations.)

Measurement noise variance. Again, the measurement noise $z_{l}[n]=\bar{h}_{l}[n]-h_{l}[n]$ (cf. (5)) cannot be observed but it can be estimated by

$$
\hat{\mathbf{z}}_{l}[n]=\tilde{\mathbf{h}}_{l}[n]-\hat{\mathbf{h}}_{l}[n \mid n-1],
$$

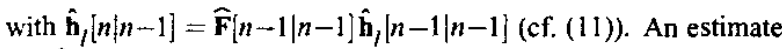
of ${ }_{z}^{2}$ is then given by

$$
\widehat{\frac{2}{z}}[n]=\frac{1}{L n M}{ }_{l=0 m=1}^{L-1} n\left\|\hat{\mathbf{z}}_{l}[m]\right\|^{2} .
$$

\section{SIMULATION RESULTS}

Simulation setup. We simulated a MIMO-OFDM system with parameters $M_{\mathrm{T}}=M_{\mathrm{R}}=4, K=64, P=16$, and $L_{\mathrm{cp}}=15$. A QPSK symbol alphabet was used. We considered two different MIMO channels that were derived from real channel data measured in an of-

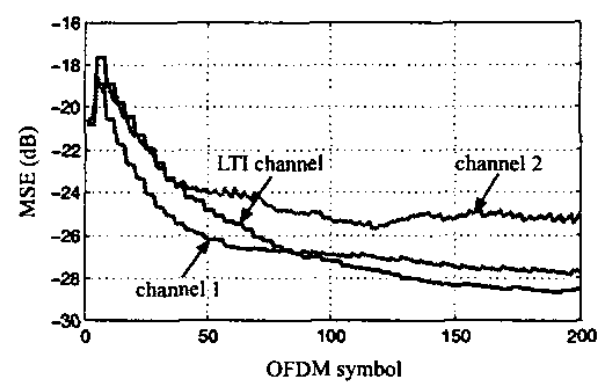

Figure 3: Convergence behavior of the proposed channel tracking scheme at an SNR of $20 \mathrm{~dB}$.

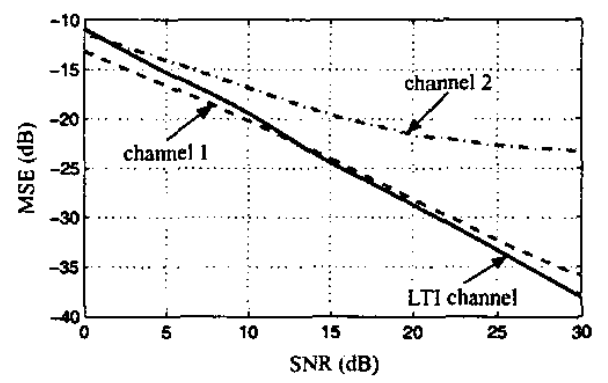

Figure 4: Normalized channel estimation MSE versuS SNR.

fice building by means of Elektrobit's PropSound channel sounder at a carrier frequency of $2.4 \mathrm{GHz}$ and with a bandwidth of about $120 \mathrm{MHz}$. The channel snapshots were measured every $37 \mathrm{~ms}$; interpolation of these snapshots by factors of 8000 and 1000 yielded time-varying channels with (estimated) maximum relative Doppler shifts $\max K=1.6 \cdot 10^{-3}$ ("channel 1 ") and $\max K=0.9 \cdot 10^{-2}$ ("channel 2"), respectively. The channel impulse responses had $L=16$ delay taps. The two channels obtained by this procedure are unrealistically fast for indoor scenarios; we used them in order to be able to demonstrate the limits of our channel tracking method. For comparison, also a synthetic time-invariant MIMO channel with $L=16$ taps ("LTI channel") was used.

The measured channel impulse responses allowed blockwise operation with blocklength $I=500$. For each block, the SNR is defined as $\begin{array}{cc}I-1 \\ n=0\end{array} \quad \begin{aligned} & K=0 \\ & k=0\end{aligned}\left\|\check{\mathbf{H}}_{n, k}\right\|_{F}^{2} /\left(I K M_{n}^{2}\right)$ (the transmit symbol vectors were normalized as $\left.\left\|\mathbf{a}_{n, k}\right\|=1\right)$.

Convergence. In Fig. 3, we show the convergence behavior of our channel tracking scheme at an SNR of $20 \mathrm{~dB}$. The normalized mean-square error (MSE) was estimated by averaging over 10 simulation runs. The MSE curves show discontinuities because the channel estimates are updated only every $M_{\mathrm{T}}=4$ OFDM symbols. It is seen that the initial convergence speed for the two time-varying channels is similar: within the first 30 OFDM symbols, the MSE decreases from $-20 \mathrm{~dB}$ to about $-25 \mathrm{~dB}$. The steady-state MSE is highest for channel 2 and lowest for the LTI channel; convergence to the steady-state MSE is faster for the time-varying channels than for the LTI channel.

MSE versus SNR. Fig. 4 depicts the steady-state MSE (estimated by averaging over 5000 OFDM symbols) versus the SNR. The MSE 


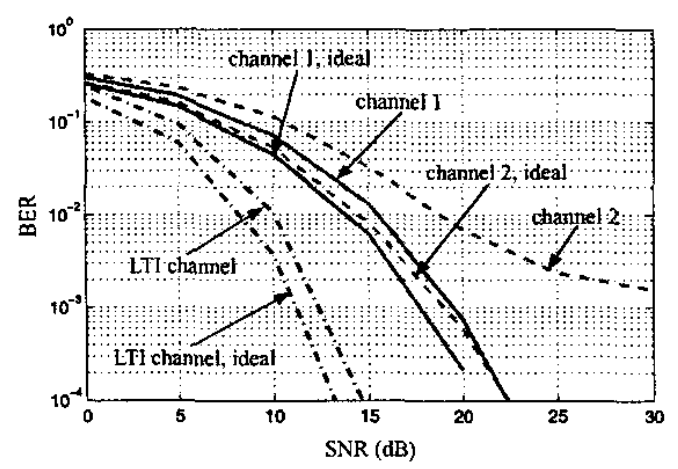

Figure 5: BER versus SNR obtained with a sphere-decoder ML receiver using estimated and ideal channel state information.

obtained for channel 1 is seen to be similar to that obtained for the LTI channel and very low, in spite of the extremely fast time variations of channel 1 . Thus, the performance of our tracking method is excellent even for very fast channels. For the (even faster) channel 2 , the MSE levels out at about $-24 \mathrm{~dB}$.

BER versus SNR. To evaluate the bit error rates (BER) that can be obtained using our channel tracking technique, we employed a simple space-frequency block precoding. Within each OFDM symbol, 96 QPSK information symbols were mapped to $(K-P) M_{\mathrm{T}}=$ 192 data symbols by applying an $8 \times 4$ precoding matrix to 24 groups of four symbols each. As precoding matrix we used four columns of an $8 \times 8$ normalized DFT matrix. To exploit frequency (delay) diversity, each group of symbols was then mapped to the four transmit antennas using two maximally separated subcarriers. At the receiver, ML decoding of each symbol group was performed by means of a sphere decoder [18] that was provided with the respective channel estimates. Furthermore, an ML decoder using the true channel coefficients (labeled "ideal") serves as a benchmark.

Fig. 5 shows that for the LTI channel and channel 1 , the BER of our receiver is close to that of the ideal receiver. This can be explained by the high accuracy of our channel tracking scheme for these channels. For the unrealistically fast channel 2, where channel tracking is less accurate, the BER is significantly larger than that of the ideal receiver, and it features an error floor at high SNR.

\section{CONCLUSIONS}

We proposed a pilot symbol assisted, adaptive channel tracking technique for MIMO-OFDM systems operating over doubly selective fading channels. Our algorithm uses an extended Kalman filter to track the channel while simultaneously providing on-line estimation of the channel's state-space parameters (state-transition matrix and noise variances). This approach is advantageous because no prior knowledge is required and spatial channel correlations are automatically exploited. Simulation results for a $4 \times 4$ system indicate that accurate channel estimation can be achieved for all practically relevant ranges of SNR and maximum Doppler frequency, and that the BER obtained with a receiver using our channel tracking scheme is close to that of an ideal receiver disposing of perfect channel knowledge. These results also confirm the validity of the state-space modeling and on-line parameter estimation approach underlying our method.

\section{ACKNOWLEDGMENTS}

The authors would like to thank D. Seethaler for contributing parts of the simulation software. They are furthermore grateful to $M$. Herdin and H. Özcelik for providing the channel measurement data.

\section{REFERENCES}

[1] G. J. Foschini and M. J. Gans, "On limits of wireless communications in a fading environment when using multiple antennas," Wireless Personal Communications, vol. 6, pp. 311-335, 1998.

[2] V. Tarokh, N. Seshadri, and A. R. Calderbank, 'Space-time codes for high data rate wireless communications: Performance criterion and code construction," IEEE Trans. Inf. Theory, vol. 44, pp. 744-765, March 1998.

[3] D. Agrawal, V. Tarokh, A. Naguib, and N. Seshadri, 'Space-time coded OFDM for high data rate wireless communications over wideband channels," in Proc. IEEE VTC-98, (Ottawa, Canada), pp. 22322236, May 1998.

[4] H. Bölcskei, D. Gesbert, and A. J. Paulraj, "On the capacity of OFDMbased spatial multiplexing systems," IEEE Trans. Conm., vol. 50, pp. 225-234, Feb. 2002.

[5] Y. Li, N. Seshadri, and S. Ariyavisitakul, "Channel estimation for OFDM systems with transmitter diversity in mobile wireless channels," IEEE J. Sel. Areas Comm., vol. 17, pp. 461-471, March 1999.

[6] Y. Li, J. H. Winters, and N. R. Sollenberger, 'MIMO-OFDM for wireless communications: Signal detection with enhanced channel estimation,"IEEE Trans. Comm., vol. 50, pp. 1471-1477, Sept. 2002.

[7] Y. Li, 'Simplifi ed channel estimation for OFDM systems with multiple transmit antennas," IEEE Trans. Wireless Comm., vol. 1, pp. 67-75, Jan. 2002.

[8] I. Barhumi, G. Leus, and M. Moonen, "Optimal training sequences for channel estimation in MIMO OFDM systems in mobile wireless channels," in Int. Zurich Seminar on Broadband Communications, (Zurich, Switzerland), pp. 441-446, Feb. 2002.

[9] D. Schafhuber, M. Rupp, G. Matz, and F. Hlawatsch, "Adaptive identifi cation and tracking of doubly selective fading channels for wireless MIMO-OFDM systems," in Proc. IEEE SPAWC-03, (Rome, italy), June 2003.

[10] C. Komninakis, C. Fragouli, A. H. Sayed, and R. D. Wesel, 'Multiinput multi-output fading channet tracking and equalization using Kalman estimation," IEEE Trans. Signal Processing, vol. 50, pp. 1065-1076, May 2002.

[11] T. Roman, M. Enescu, and V. Koivunen, 'Time-domain method for tracking dispersive channels in MIMO OFDM systems," in Proc. IEEE ICASSP-2003, vol. 4, (Hong Kong), pp. 393-396, April 2003.

[12] R. Negi and J. Cioff, 'Pilot tone selection for channel estimation in a mobile OFDM system," IEEE Trans. Consumer Electron., vol. 44, pp. 1122-1128, Aug. 1998.

[13] L. Ljung, "Asymptotic behavior of the extended Kalman filter as a parameter estimator for linear systems," IEEE Trans. Autom. Contr:, vol. 24, pp. 36-50, Feb. 1979.

[14] T. Kailath, Linear Systems. Englewood Cliffs (NJ): Prentice Hall, 1980.

[15] J. W. Brewer, 'Kronecker products and matrix calculus in system theory," IEEE Trans. Circuits and Systems, vol. CAS-25, pp. 772-781, Sept. 1978.

[16] K. A. Myers and B. D. Tapley, "Mdaptive sequential estimation with unknown noise statistics," IEEE Trans. Autom. Contr., vol. 21, pp. 520-523, Aug. 1976.

[17] R. K. Mehra, "On the identifi cation of variances and adaptive Kalman filtering," IEEE Trans. Autom. Contr., vol. 15, pp. 175-184, April 1970.

[18] M. Damen, A. Chkeif, and J. Belfi ore, "Lattice code decoder for spacetime codes," IEEE Comm. Letters, vol. 4, pp. 161-163, May 2000. 